ESAIM: PROCEEDINGS AND SURVEYS, June 2017, Vol. 56, p. 136-138

S. Crépey, M. Jeanblanc and A. Nikeghbali Editors

\title{
LES DÉBUTS DE LA THÉORIE DU GROSSISSEMENT DE FILTRATIONS
}

\author{
T. JEULIN ${ }^{1}$
}

\begin{abstract}
From the presentation of T. Jeulin, on May 3rd 2016 at Institut Henri Poincaré, Paris.
Résumé. D'après les notes d'exposé de T. Jeulin du 3 mai 2016 à Paris, Institut Henri Poincaré.
\end{abstract}

\section{Contexte}

Dans les années 70, les thèmes de recheche en probabilités portaient sur divers domaines, principalement

(1) la théorie générale des processus (Dellacherie, Meyer, Azéma et al.)

(2) les processus de Markov (aspect "processus droits" dans la lignée de Meyer, Ray, Blumenthal-Getoor, le livre de Sharpe ne paraîtra qu'en 1988)

(3) le calcul stochastique (sous tous ses aspects, y compris espaces $\mathrm{H}^{p}$ et EDS)

(4) les problèmes de martingales (Stroock-Varadhan pour les diffusions, Jacod pour des versions plus abstraites)

(5) Girsanov ; Stricker ; Bichteler-Dellacherie, mesures de Föllmer.

(6) Ou encore contrôle, chaînes de Markov, convergence de processus, etc ...

L'article d'Azéma (1972) où il étudie en particulier les fins de prévisibles, est précurseur dans le domaine de grossissement de filtration.

A cette époque, il n'y avait pas de motivation financière (les articles de Merton, tout comme le modèle de Black-Scholes-Merton m'étaient complètement inconnus ; je n'ai eu connaissance de leur existence qu'en étudiant - avec d'autres - les articles de Harisson et Pliska, vers 1983).

Il faut se rendre compte que la diffusion de l'information "mathématique" était alors beaucoup plus confidentielle que maintenant et relevait des relations personnelles (c'était donc plutôt restreint) ou de la lecture en bibliothèque des lourds volumes des Math. Reviews ou du Zentralblatt.

L'informatisation a bouleversé tout cela (et on peste aujourd'hui quand un article cité n'est pas accessible depuis son ordinateur personnel ...). De même TeX ou LaTex n'avaient pas atteint tous les mathématiciens (il y avait encore des secrétaires qui s'occupaient de la frappe des documents, sur de vraies machines à écrire, et de leur reproduction à l'aide de stencils ...) ; et certains étaient plus fiers de la qualité de leurs "macros" que du contenu scientifique du texte produit.

\section{LES DÉBUTS}

Mon initiation à ce domaine s'est faite à travers une version préliminaire du texte de M. T. Barlow, dans le train, avec M. Yor, en revenant du Séminaire de Probabilités qui avait eu lieu à Rennes, en 1977 ; c'était aussi

\footnotetext{
1 Laboratoire de Probabilités et Modèles aléatoires Mathématiques, Université Paris 7 - Denis Diderot, 2, Place Jussieu, 75251 Paris Cedex 05.
}

(C) EDP Sciences, SMAI 2017 
pour moi le début d'une collaboration fructueuse avec Marc) ; le but était d'étudier, via la théorie générale des processus les décompositions de Millar des trajectoires des processus de Markov.

Un autre article "fondateur" est celui de K. Itô de 1976, découvert plus tard, où il s'agit d'étendre le domaine de définition de l'intégrale stochastique.

Nous partions de l'idée que l'on pouvait appliquer la théorie générale des processus et le calcul stochastique (et donc le grossissement de filtrations qui en est un avatar) pour des problèmes explicites ; j'y reviendrai en commentant la bibliographie..

Pour un historique, voir l'introduction du chapitre 6 du livre de P. Protter.

Je noterai quelques points fondamentaux :

- dans le grossissement "progressif" avec une variable honnête, on ajoute seulement à la tribu prévisible les ensembles $[0, L]$ et $] L, \infty[$;

- le grossissement "initial" de la filtration $\mathcal{F}$ par une variable $Z$ apparaît une version élaborée de la formule de Bayes ; il est important de contrôler le processus optionnel des lois conditionnelles $\left(\mathbb{P}\left[Z \in . \mid \mathcal{F}_{t}\right]\right)_{t \geq 0}$. Un exemple frappant en est le résultat de Jacod (Grossissement initial, hypothese (H'), et théorème de Girsanov. L.N. Math. 1118, 1985) où ces lois sont toutes absolument continues par rapport à une mesure fixe. Ne rentre pas dans ce cadre l'exemple suivant : $T$ est un temps d'arrêt totalement inaccessible, de compensateur $A$ et $Z=A_{\infty}$.

Je relirais volontiers la thèse de Song (1987)

Après sont venus le calcul de Malliavin (et, au début, tout ce qui faisait référence à l'hypoellipticité dans le recherche de densités pour les solutions d'eds), les probabilités quantiques, les probabilités non commutatives, les processus à valeurs mesures, les Mathématiques financières (avec, en particulier, les problèmes de non-arbitrage), la théorie des matrices aléatoires (et les questions d'universalité), etc ... La théorie générale des processus et le calcul stochastique peuvent être des outils dans certains des domaines cités, pas dans tous évidemment!

\section{BIBLIOGRAPHIE NON EXHAUSTIVE (JUSQU'EN 1985)}

Azema J. : Quelques applications de la théorie générale des processus-I. Inv. Math. 18, 1972.

BARLOW M.T. : Study of a filtration expanded to include an honest time. Z.f.W. 44, 1978 (le déclencheur).

Brémaud P., Yor M. : Changes of filtrations and of probability measures. Z.f.W. 45, 1978 (apparition de l'hypothèse $(\mathrm{H})$ : les $\mathcal{F}$-martingales restent des $\mathcal{G}$-martingales, qui nécessitera le passage à ( $\mathrm{H}$ ') dans le cas plus général les $\mathcal{F}$-semimartingales restent des $\mathcal{G}$-semimartingales ; plaisanterie sur (Gr) pour "grossissement" et rayon diététique des librairies ... )

Chaleyat-Maurel M. : Exemples de grossissement gaussien de la filtration Brownienne. Ann. Sc. ClermontFerrand M-71, 1982.

Dellacherie C., Meyer P.A. : A propos du travail de Yor sur le grossissement des tribus. Sem. Prob. 12, L.N. Math. 649, Springer, 1978.

Grossissements de filtrations : exemples et applications. Séminaire de Calcul Stochastique, 1982/83, Université Paris Vl (organisé en commun avec M. Yor). L.N. Math. 1118, Springer 1985.

En particulier :

- Chaleyat-Maurel M., Jeulin T. : Grossissement Gaussien de la filtration brownienne.

- JACOD J. : Grossissement initial, hypothèse (H'), et théorème de Girsanov.

- JEULin T. : Temps local et théorie du grossissement, application de la théorie du grossissement à l'étude des temps locaux browniens (Les théorèmes de Ray-Knight-Walsh concernant le mouvement Brownien peuvent se lire sur la formule de Tanaka à condition d'écrire cette formule dans une filtration appropriée).

- Yor M. : Grossissement de filtrations et absolue continuité de noyaux.

- Yoeurp C. : Théorème de Girsanov généralisé et grossissement d'une filtration.

Iтô K. : Extension of stochastic integrals. Proc. Intern. Symp. SDE, Kyoto, 1976. 
JACOD J. : Calcul stochastique et problèmes de martingales. L.N. Math. 714. Springer 1979 (en particulier Ch. 9 : changements de filtration).

Jeulin T., Yor M. : Nouveaux résultats sur le grossissement des tribus. Ann. Sci. École Norm. Sup. 11, 1978.

JEULIN T. : Comportement des semi-martingales dans un grossissement de filtration. Z. f. W. 52, 1980.

JEULin T. : Grossissement de filtrations et applications. L.N. Math. 833, Springer 1981 (c'est le contenu de la thèse, soutenue en février 1980).

MeYer P.A. : Les résultats de Jeulin sur le grossissement des tribus. Sem. Prob. 14, L.N. Math. 784, Springer, 1980 .

Millar P.W. : Path decomposition for Markov processes. Ann. Prob. 6, 1978.

STRICKER C. : Quasimartingales, martingales locales, semimartingales et filtration naturelle. ZfW 39, 1977.

Yor M. : Application d'un lemme de T. Jeulin au grossissement de la filtration brownienne. Sem. Prob. 14, L.N. Math. 784, 1980.

Yor M. : Grossissement d'une filtration et semimartingales - Théorèmes généraux. Sem. Prob. 12, L.N. Math. 649, Springer, 1978.

Sans oublier

Föllmer H., Imkeller P. : Anticipation cancelled by a Girsanov transformation - A paradox on Wiener space. Ann. I.H.P. B, 29, 1993.

Protter P. : Stochastic integration and differential equations. Springer, 2004 (Chapitre 6). 